\title{
Two cases of less invasive treatment for radicular cyst in primary dentition
}

\author{
Atsuo Sakurai*1, Yoshitaka Taniguchi*2, Tetsuyuki Hayashibara*3, Sachie Senzui*1, \\ Hiromi Homma*4, Tatsuo Hashida*5, Takashi Ooshima*4 and Seikou Shintani*1 \\ *1 Department of Pediatric Dentistry, Tokyo Dental College \\ 1-2-2 Masago, Mihama-ku, Chiba 261-8502, JAPAN \\ *2 The First Department of Oral and Maxillofacial Surgery, Osaka University Graduate School of Dentistry \\ 1-8 Yamada-oka, Suita, Osaka 565-0871, JAPAN \\ *33 Hayashibara Dental Clinic \\ 6-7-3 Higashifukuhara, Yonago, Tottori 682-0802, JAPAN \\ ${ }^{*}$ Department of Pediatric Dentistry, Osaka University Graduate School of Dentistry \\ 1-8 Yamada-oka, Suita, Osaka 565-0871, JAPAN \\ *5 Department of Dentistry, Sakai City Health Promotion and Welfare Center, Chinugaoka Clinic \\ 3-128-4 Kyowacho, Sakai, Osaka 590-0822, JAPAN
}

\begin{abstract}
Radicular cysts in the primary dentition are rare, with such cysts most commonly occurring in the permanent dentition. Pulpal treatment for teeth affected by dental caries has been suggested as a causative factor of radicular cysts. We report two cases of radicular cysts occurring in the primary dentition after pulpectomy. Marsupialization of the cystic lesion was performed in both cases, resulting in uneventful eruption of the successive permanent tooth. A resin appliance in one case and antibiotic ointment-impregnated gauze only in the other were used to fill and promote healing of the cystic cavity. The results suggest that this approach is effective in inducing complete healing of radicular cysts in the primary dentition and avoiding the formation of residual cysts. Furthermore, use of antibiotic-impregnated gauze appears to offer an easier option than other reported methods in the management of radicular cysts.
\end{abstract}

Key words

Primary dentition,

Radicular cyst,

Tooth eruption,

Treatment protocols

\section{Introduction}

Radicular cysts originate in low-grade irritation of periapical tissue, which leads to proliferation of the epithelial rests of Malassez. Radicular cysts are often caused by pulp necrosis associated with dental caries or trauma ${ }^{1,2)}$, and although common in the permanent dentition, are rare in the primary dentition. According to some reports, radicular cysts in the primary dentition occupy only $0.5-3.3 \%$ of the total number ${ }^{1,3)}$. The reason for this is unclear, although it has been suggested that this is due to the shorter time that the primary teeth are present in the oral cavity than the permanent teeth ${ }^{4}$. When pulpal

Received on October 1, 2010

Accepted on February 15, 2011 lesions occur following the development of periodontitis, they tend to be interradicular, rather than apical. Moreover, periapical infections in the primary dentition appear to drain more readily than those in the permanent dentition because the bone structures have yet to be fully formed in early childhood ${ }^{5}$. According to some earlier reports, most radicular cysts associated with the primary teeth have occurred following endodontic treatment involving pulpal therapeutic agents, and rapid growth of the cyst has been observed in some cases ${ }^{5-7)}$. Radicular cysts are often asymptomatic in the early stages, unless acute inflammation is present, and can be detected only by radiographic examination ${ }^{8)}$.

We report two cases of radicular cysts arising in the primary dentition. Less invasive procedure was carried out in both cases, including extraction of the 


\section{A}

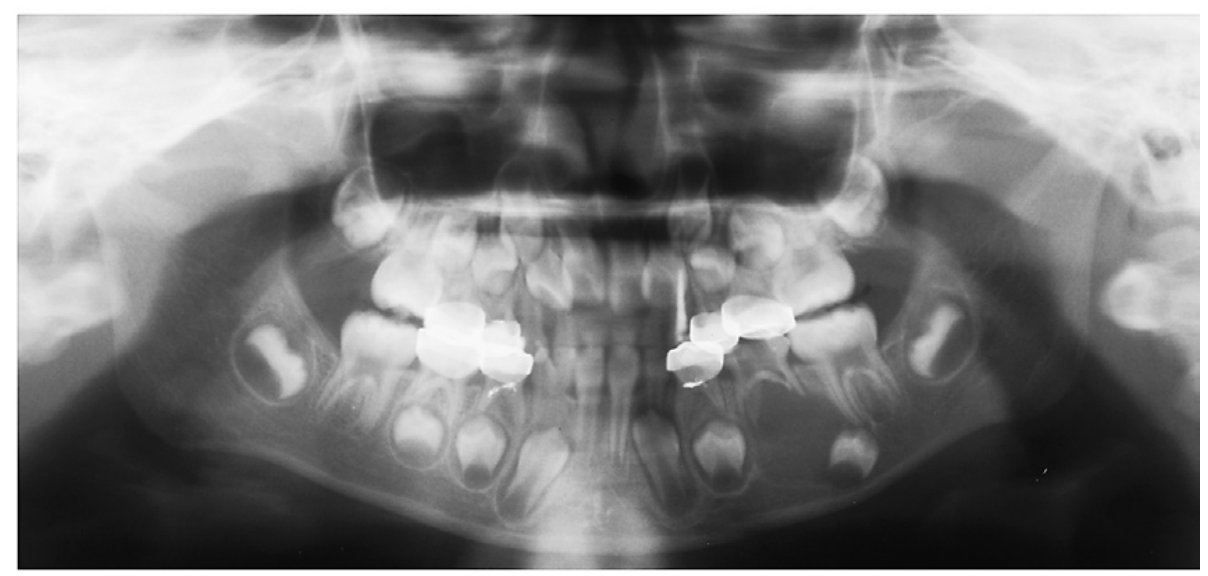

B

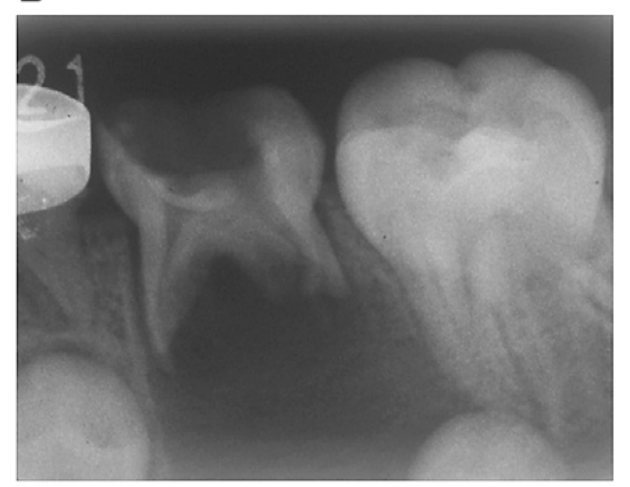

C

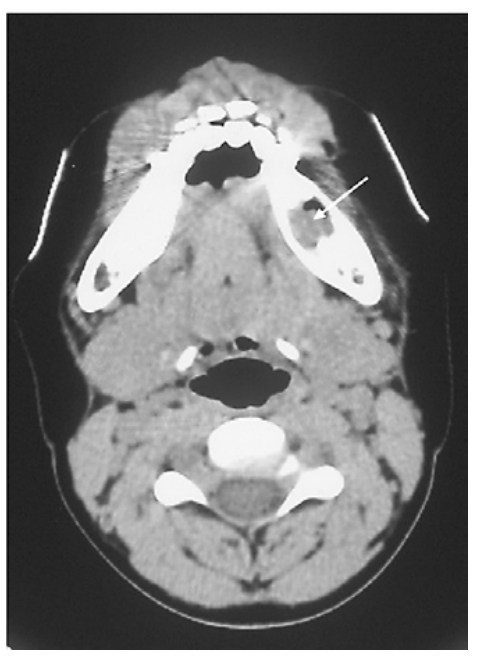

Fig. 1 Radiographs of Case 1 at first visit. (A) Dental panoramic radiograph. Radiolucent area was associated with root of mandibular left primary second molar. (B) Periapical radiograph of affected tooth. (C) Horizontal CT image of cystic lesion. Arrow indicates radiolucent area containing liquid component.

affected primary tooth followed by marsupialization of the cyst. The subsequent courses of recovery are discussed.

\section{Case Reports}

\section{Case 1}

A Japanese girl aged 6 years 11 months visited the Pediatric Dental Clinic of Osaka University Dental Hospital for examination of gingival swelling. Clinical inspection revealed gingival swelling in the region of the primary mandibular left molars, and marked mobility was noted at the primary mandibular left second molar. She had had many carious lesions, especially in the primary molars, and had received stainless steel crown restoration following pulp treatment. Approximately 30 months before first presenting at our hospital, she had undergone a pulpectomy and therapy with agents containing calcium hydroxide and iodoform (Vitapex, Neo Dental Chemical Products, Tokyo, Japan) for a carious lesion in the primary mandibular left second molar. Although she had been symptomless for a long time, she complained of slight tenderness at the time of her visit. Dental panoramic tomography and periapical radiography revealed a well-defined and rounded radiolucent area $25 \mathrm{~mm}$ in diameter associated with the root of the primary mandibular left second molar (Figs. 1A, B). The successive permanent mandibular left second premolar was dislocated inferiorly and posteriorly. Computerized tomography $(\mathrm{CT})$ also revealed a radiolucent lesion 


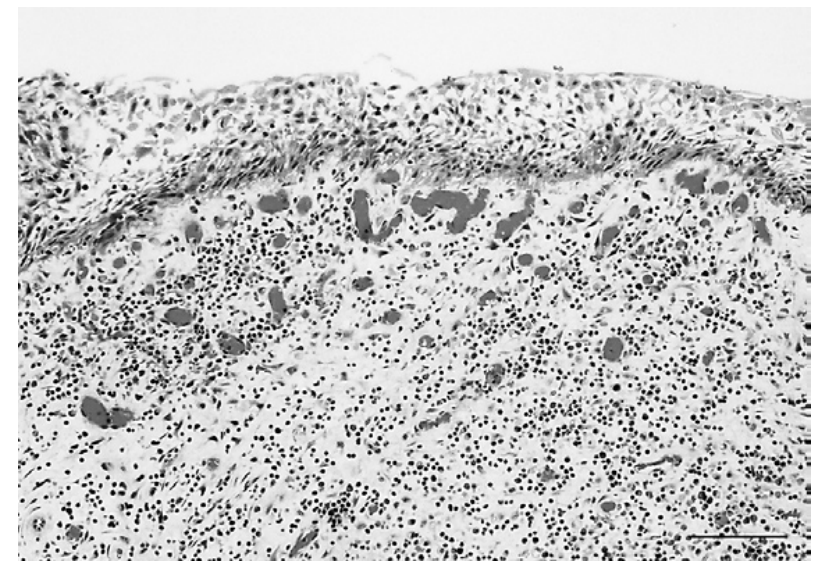

Fig. 2 Histological image of biopsy specimens from Case 1. Nonkeratinized, stratified squamous epithelium and dense inflammatory cells observed. Bar: $200 \mu \mathrm{m}$.

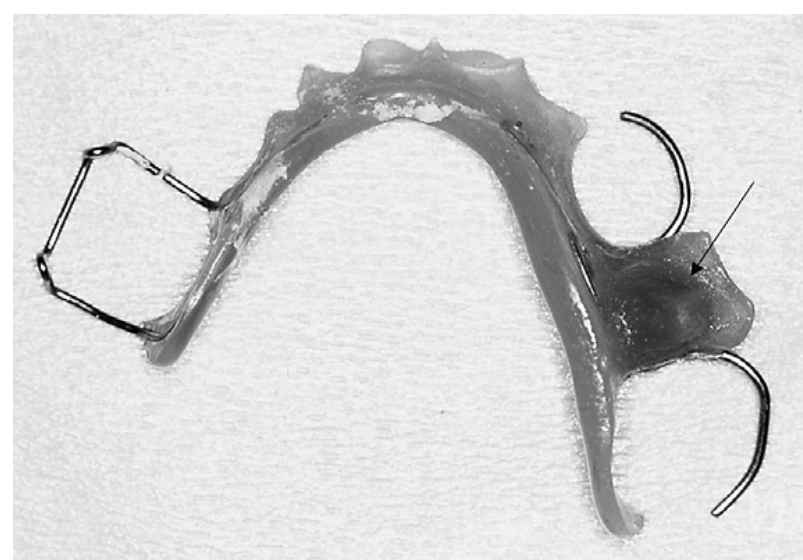

Fig. 3 Mucosal surface view of removable appliance. Arrow indicates resin projection penetration of lesion.

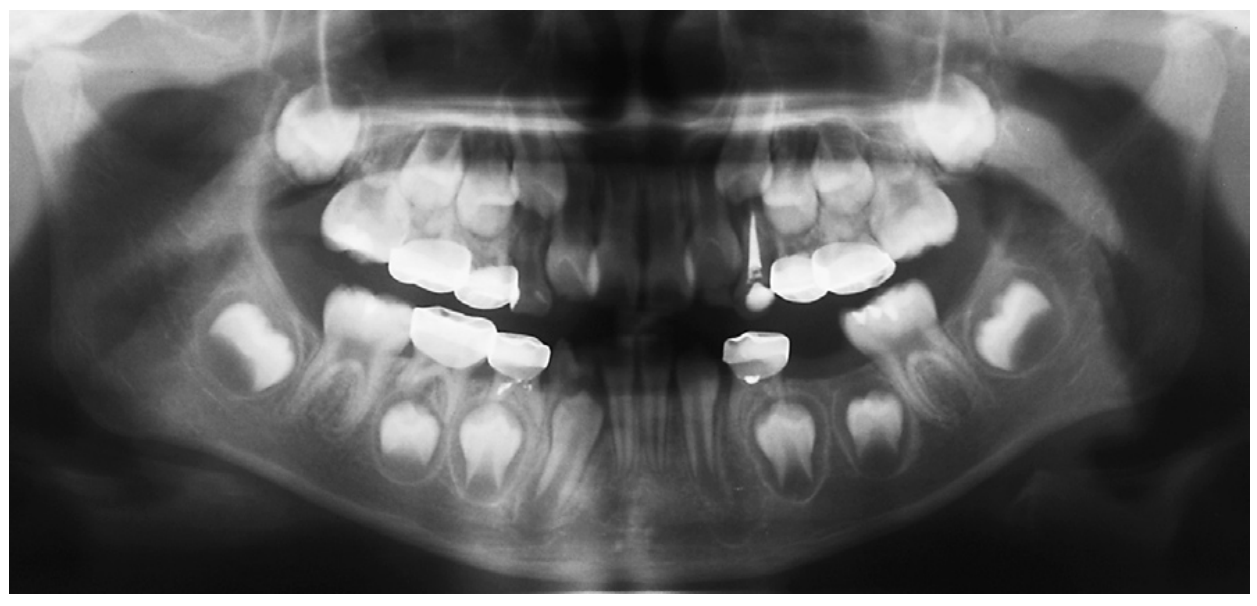

Fig. 4 Dental panoramic radiograph of Case 1 at 12 months after extraction of mandibular left primary second molar. Successive tooth was observed at putative original position.

in the affected region, and the Hounsfield number of internal mean density was approximately 30, indicating a liquid component (Fig. 1C). Clinical and radiographic findings suggested that the lesion was a radicular cyst arising from the primary mandibular left second molar, rather than a radicular granuloma.

The lesion was surgically treated by extraction of the primary mandibular left second molar followed by marsupialization for decompression. Part of the affected tissue below the extracted molar was dissected and prepared for microscopic examination. The specimen showed nonkeratinized, stratified squamous epithelium and connective tissue with intense infiltration of inflammatory cells, indicating the lesion to be a cyst (Fig. 2). The tooth socket and cystic cavity were filled with gauze impregnated with Achromycin ointment for 2 weeks. A removable appliance with a resin projection for penetration of the cystic cavity was then fitted to prevent epithelial overgrowth (Fig. 3). This appliance was adjusted at each subsequent visit, and the cystic lesion gradually decreased in size, finally closing at 3 months after initiation of treatment. Thereafter, the resin appliance was used as a space retainer to prevent mesial inclination of the permanent mandibular left first molar. The germ of the permanent mandibular left second premolar, which was examined monthly by visual inspection and radiography, gradually recovered its normal position (Fig. 4). The premolar 
A

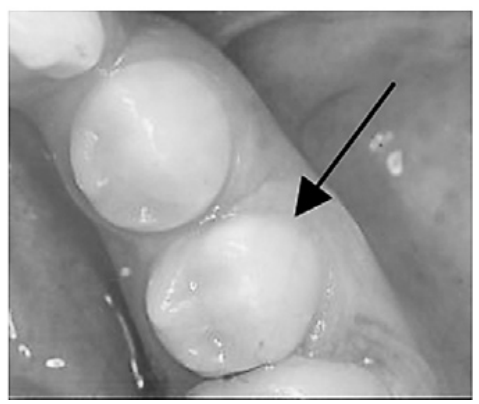

B

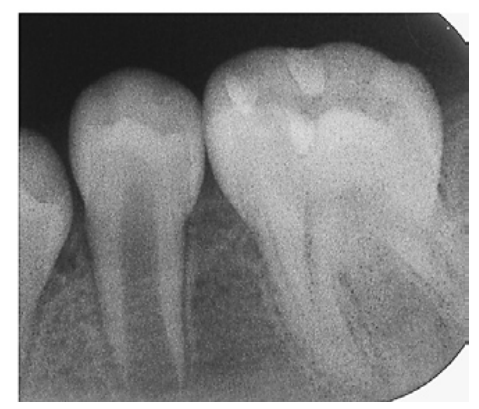

Fig. 5 Clinical and radiographic appearances of affected lesion at 37 months after operation. (A) Clinical view. Mandibular left second premolar erupted with no occurrence of residual cyst (An arrow). (B) Radiographical view. Radiolucent area disappeared.

\section{A}

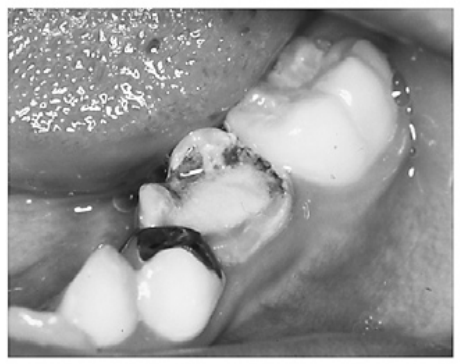

B

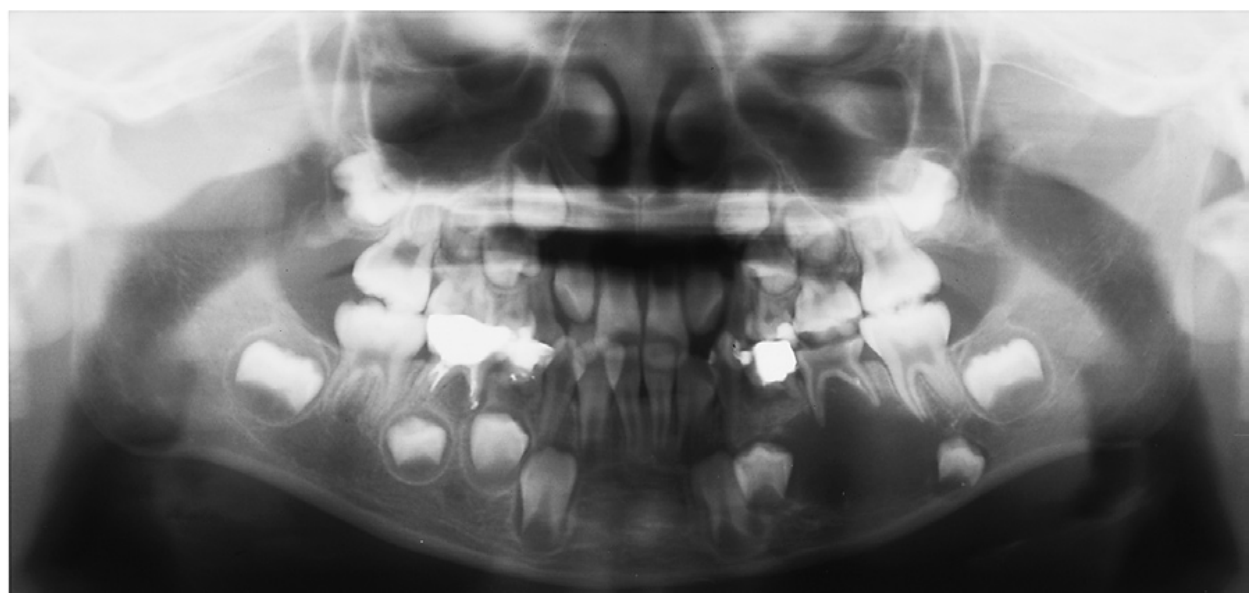

Fig. 6 Clinical and radiographic appearances of Case 2 at first visit. (A) Clinical view. Pulp chamber was opened and filled with cotton. (B) Dental panoramic photograph. Radiolucent area was observed immediately below root of mandibular left primary second molar.

erupted with no further complications apart from slight enamel hypoplasia when she reached 10 years of age (Figs. 5A, B).

\section{Case 2}

A Japanese girl aged 8 years 2 months was referred to our hospital by her general dentist. She had experienced some pain in the region of the primary mandibular left molars on eating. Clinical examination revealed mild tooth mobility in the primary mandibular left second molar (Fig. 6A). Although her general dentist had opened the pulp chamber, 


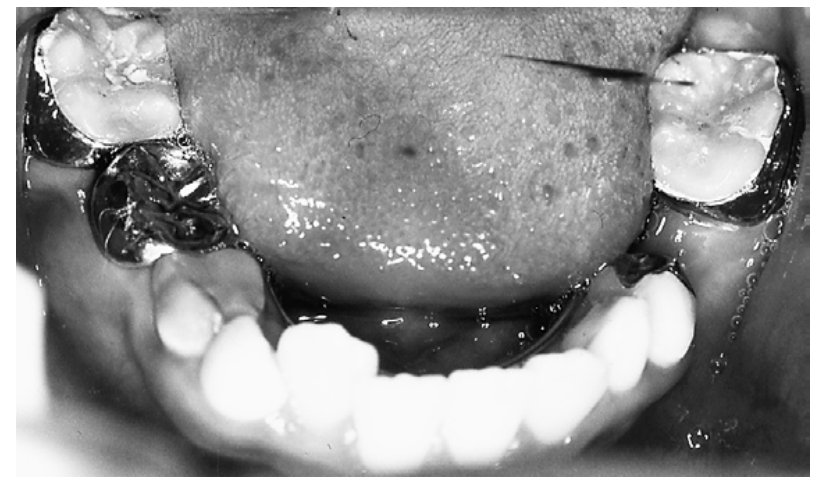

Fig. 7 Clinical view of Case 2 at 10 months after extraction of affected teeth. Space retainer was applied.

no evidence of pus drainage from the root canal was observed. The tooth had been treated with a pulpectomy 3 years prior to her visit. Other than pain on eating, she reported no other symptoms. Dental panoramic tomography revealed a radiolucent area $30 \mathrm{~mm}$ in diameter associated with the root of the primary mandibular left second molar (Fig. 6B). The successive permanent mandibular left second premolar and adjacent first premolar showed marked dislocation.

The primary mandibular left second molar was extracted and a cyst wall associated with the root of the affected tooth fenestrated. Histopathological examination of the affected tissue below the extracted tooth revealed nonkeratinized epithelium and connective tissue with infiltrating inflammatory cells (Data not shown). The cystic cavity was filled with gauze impregnated with Achromycin ointment, which was changed weekly over a period of 40 days. The cystic cavity reduced in size and the lesion closed 40 days after marsupialization. Thereafter, a fixed space retainer was applied (Fig. 7). Radiography revealed restitution of the permanent mandibular left second premolar and first premolar germs (Fig. $8)$. The premolars erupted normally when she was 12 years old.

\section{Discussion}

Radicular cysts in the primary dentition may be misdiagnosed as periapical granulomas of the primary teeth or follicular cysts of their permanent

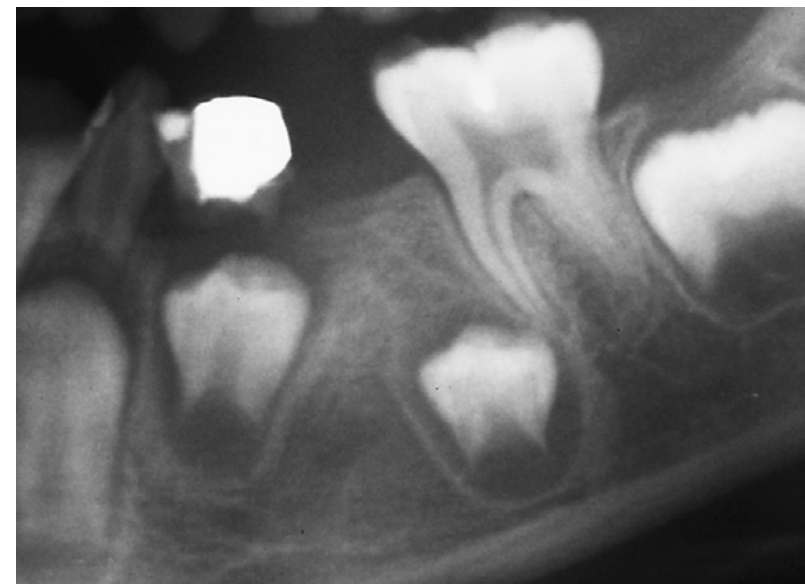

Fig. 8 Enlarged dental panoramic radiograph of Case 2 at 10 months after operation. Marked restitution of mandibular left first and second premolars observed.

successors $^{7)}$, and it is particularly difficult to distinguish them radiographically. In the present cases, a diagnosis was reached after histopathological examination confirmed the presence of a cystic epithelial lining. An accurate diagnosis may further be complicated due to the roots of the primary molars being closely situated to the region of the permanent teeth germs. Therefore, we obtained surgical confirmation of a diagnosis of radicular cyst, rather than relying on radiographic findings alone. Radiography, however, revealed that the cystic wall was connected to the root tip, and that the follicle of the permanent tooth germ was distinct from the cystic wall of the affected tooth.

A previous report summarized several criteria to support the diagnosis of a radicular cyst ${ }^{9)}$, which included a non-vital tooth, mandibular buccal cortical expansion and a painless lesion associated with the primary tooth. Histopathological confirmation of a cystic epithelial lining is also considered important $t^{9)}$. All these criteria were fulfilled in both the present cases. Moreover, it was found that i) the radiolucent lesions were associated with the primary teeth and did not involve the successive permanent teeth; ii) the successive permanent teeth were displaced; and iii) there was no association with the successive permanent teeth, all of which were confirmed on extraction of the affected teeth and subsequent marsupialization. All of this was consistent with established criteria ${ }^{7,9-11)}$. Furthermore, our present findings agree with those of another report which indicated that all radiolucent 
lesions of more than $20 \mathrm{~mm}$ in diameter associated with the primary teeth suggested a diagnosis of radicular cyst ${ }^{12)}$.

In this report, both patients had had severe caries of the primary teeth which was treated with pulpectomy. The cyst in both cases originated from the primary mandibular left second molar and radiographic appearances were remarkably similar. The primary mandibular second molar was reported as the site of highest occurrence of radicular cysts in the primary teeth, as this tooth is the most frequently affected by dental caries ${ }^{8,13)}$. It is controversial as to whether the treatment of dental pulp is a causative factor in the development of radicular cysts of the primary teeth. According to some reports, many cases of radicular cysts associated with the primary molars occurred after various kinds of pulp treatment $^{5-7)}$. In contrast, other studies suggested that radicular cysts were not related to the treatment of dental pulp ${ }^{2,8}$. In addition, after analyzing primary teeth and finding radiolucent lesions ranging from 4 to $15 \mathrm{~mm}$ in diameter, Mass et al. insisted that radicular cysts in the primary molars were not rare, but merely overlooked ${ }^{2}$. In such cases, the only treatment given may be root canal therapy or extraction. However, radicular cysts of the primary teeth involving radiolucent areas of more than $20 \mathrm{~mm}$ in diameter appear to be rare, and it is possible that pulpal therapeutic agents cause rapid growth of cystic lesions ${ }^{7}$. Endodontically treated primary teeth should be observed until they are shed.

The cystic lesions in our report were treated with marsupialization, rather than enucleation of the entire cystic wall. In both cases, the successive permanent teeth erupted uneventfully, indicating that the procedure used was appropriate for obtaining a good prognosis. Such conservative treatment is less invasive, involves less danger of damaging the permanent tooth germ and is more acceptable to the patients and their parents/guardians than enucleation $^{4)}$. In some earlier reports on conservative treatment for radicular cysts in the primary dentition, resin appliances were used to promote decompression of the cystic lesion and prevent the accumulation of food in the cystic cavity ${ }^{4,14)}$. Furthermore, it is crucial to prevent epithelial tissue covering the superior border of the cystic cavity only, as such poor healing may lead to the formation of a residual cyst ${ }^{5)}$. However, we used the resin removable appliance for just 3 months in one case and treatment with only antibiotic-impregnated gauze for 40 days in the other case, unlike in previous reports where resin appliances were used for 6 or 16 months $^{4,14)}$. Although the cystic cavities in the present cases showed a relatively fast reduction in size compared with that observed in earlier reports, no covering by epithelial tissue or development of residual cysts was observed. More cases involving treatment with antibiotic-impregnated gauze need to be observed to determine whether this method is always applicable. However, this procedure appears to offer a promising approach to inhibiting the formation of residual cysts and inducing the complete healing of radicular cysts. Furthermore, postoperative follow-up examinations are also important until the successive permanent teeth erupt. Therefore, the full co-operation of the patients and their parents/guardians is needed.

\section{Acknowledgments}

The authors wish to thank Associate Professor Jeremy Williams, Department of Biomedical Information and Communications, Oral Health Science Center, Tokyo Dental College, Tokyo, Japan, for his assistance with the English of this manuscript.

\section{References}

1) Lustmann, J. and Shear, M.: Radicular cysts arising from deciduous teeth. Review of the literature and report of 23 cases. Int J Oral Surg 14: 153-161, 1985.

2) Mass, E., Kaplan, I. and Hirshberg, A.: A clinical and histopathological study of radicular cysts associated with primary molars. J Oral Pathol Med 24: 458-461, 1995.

3) Shear, M.: Cysts of the Oral Region. 3rd ed. Wright, Oxford, 1992, pp.136-162.

4) Delbem, A.C., Cunha, R.F., Vieira, A.E. and Pugliesi, D.M.: Conservative treatment of a radicular cyst in a 5-year-old child: a case report. Int J Paediatr Dent 13: 447-450, 2003.

5) Caldwell, R.E., Freilich, M.M. and Sandor, G.K.: Two radicular cysts associated with endodontically treated primary teeth: rationale for long-term followup. Ont Dent 76: 29-33, 1999.

6) Grundy, G.E., Adkins, K.F. and Savage, N.W.: Cysts associated with deciduous molars following pulp therapy. Aust Dent J 29: 249-256, 1984.

7) Takiguchi, M., Fujiwara, T., Sobue, S. and Ooshima, T.: Radicular cyst associated with a primary molar following pulp therapy: a case report. Int J Paediatr Dent 11: 452-455, 2001.

8) Nagata, T., Nomura, J., Matsumura, Y., Yanase, S., Fujii, T., Oka, T., Uno, S. and Tagawa, S.: Radicular 
cyst in a deciduous tooth: a case report and literature review. J Dent Child (Chic) 75: 80-84, 2008.

9) Gandhi, S. and Franklin, D.L.: Presentation of a radicular cyst associated with a primary molar. Eur Arch Paediatr Dent 9: 56-59, 2008.

10) Hashida, T., Hiranuma, H., Fujishita, M., Ooshima, T. and Fuchihata, H.: Radicular cyst of primary teeth: Report of two cases. J Clin Pediatr Dent 17: 171-173, 1993.

11) Ramakrishna, Y. and Verma, D.: Radicular cyst associated with a deciduous molar: A case report with unusual clinical presentation. $J$ Indian Soc Pedod Prev Dent 24: 158-160, 2006.

12) Goaz, P. and White, S.: Oral Radiology: Principles and Interpretation. 3rd ed. Wright, St. Louis, 1994, pp.518-533.

13) Hill, F.J.: Cystic lesions associated with deciduous teeth. Proc Br Paedod Soc 8: 9-12, 1978.

14) Johann, A.C., Gomes Cde, O. and Mesquita, R.A.: Radicular cyst: a case report treated with conservative therapy. J Clin Pediatr Dent 31: 66-67, 2006. 